\title{
Morphology Correlation to the Superhydrophobicity in an Organic Thin Film on Rough Al
}

\author{
Sili Ren ${ }^{1,2,3,4}$, Shengrong Yang ${ }^{2}$, Ya-Pu Zhao ${ }^{3}$, Xudong Xiao ${ }^{1}$, Tongxi Yu ${ }^{4}$ \\ 'Department of Physics, Hong Kong University of Science and Technology, Hong Kong, \\ China; \\ ${ }^{2}$ State Key Laboratory of Solid Lubrication, Lanzhou Institute of Chemical Physics, Chinese \\ Academy of Sciences, Lanzhou 730000, China; \\ ${ }^{3}$ State Key Laboratory of Nonlinear Mechanics, Institute of Mechanics, Chinese Academy of \\ Sciences, Beijing 100080, China \\ ${ }^{4}$ Department of Mechanical Engineering, Hong Kong University of Science and Technology, \\ Hong Kong, China;
}

\begin{abstract}
A novel superhydrophobic ultra-thin film with contact angle of $166^{\circ}$ was prepared by stearic acid (STA) chemically adsorbed onto the polyethyleneimine (PEI) coated aluminum wafer. Composite interface between the water droplet and the rough needle-like surface was attributed to responsible for the super water-repellent property. It was found that close relationship existed between the surface morphology and the contact angle. The ultra-thin film has weak contact angle hysteresis with the advancing and receding contact angle about $168^{\circ}$ and $156^{\circ}$, respectively. However, if the water droplet was placed onto the surface keeping static for a long times, the contact mode might be changed from composite interface into noncomposite interface, and then generate large angle hysteresis.
\end{abstract}

Keywords: Superhydrophobicity, contact angle, morphology, AFM

\section{Introduction}

Wettability is one of the important properties of solid surfaces from both theoretical and practical aspects. ${ }^{[1-3]}$ For example, The fast developing micro electro-mechanical systems (MEMS), are known for their superior performance and low unit cost. ${ }^{[4]}$ However, the large surface-area-to-volume ratios brought the adhesive and frictional problems into these systems. ${ }^{[5,6]}$ In order to alleviate these adhesive related problems, both the topography and the chemical composition of the contacting surfaces must be controlled to produce hydrophobic surfaces.
Surfaces with superhydrophobic properties were highly paid attention. Several typical methods of preparing such superhydrophobic surfaces were reported in recent years ${ }^{[1,7-10]}$. All the methods have a common characteristic that they were obtained through a combination of enough surface roughness and hydrophobic materials. In other words, both surface geometrical structure and chemical composition control the wettability of the solid surface.

Branched polyethyleneimine $\{\mathrm{PEI}$, $\left.-\left[\mathrm{C}_{2} \mathrm{H}_{5} \mathrm{NHC}_{2} \mathrm{H}_{5} \mathrm{~N}\left(\mathrm{C}_{2} \mathrm{H}_{5} \mathrm{NH}_{2}\right) \mathrm{C}_{2} \mathrm{H}_{5} \mathrm{NH}\right]_{\mathrm{n}^{-}}\right\}$with primary and secondary amino groups can be readily modified by phosgene, thiophosgene, cyanuric chloride and glutaraldehyde. ${ }^{[1,12]}$ In this paper, we report a novel ultra-thin film with 
superhydrophobic properties, prepared by stearic acid chemically adsorbed onto the PEI-coated rough $\mathrm{Al}$ surface, with emphasis on the correlation between the surface morphology and the contact angles and the effects of times on the contact angle hysteresis.

\section{Experimental Section}

Polished aluminum wafer was used as the substrate, which was first boiled in water from 0 to 5 minutes ( 0 minutes means fresh polished $\mathrm{Al}$ substrate without boiling in water was used) to roughen the $\mathrm{Al}$ surfaces. A layer of PEI was first formed on the $\mathrm{Al}$ substrates by immersing into a dilute aqueous solution of polyethyleneimine (PEI) of $0.2 \mathrm{wt} \%$ for 15 minutes. After rinsing with ultra-pure water, the PEI-coated Al substrates were then put into a dilute solution of STA and DCCD mixture in $n$-hexane. After reacting for $24 \mathrm{~h}$, STA monolayer was produced on the PEI coating. We have confirmed that a covalent amide bond was formed between the carboxylic group and the primary or secondary amine groups. ${ }^{12}$

Contact angles for water was measured with a contact-angle goniometer (Model 100-00; Rame-hart inc, USA). The AFM surface topographies were observed on a home-made atomic force microscope with an RHK electronic controller (RHK Technology, Rochester Hills, MI).

\section{Results and discussion}

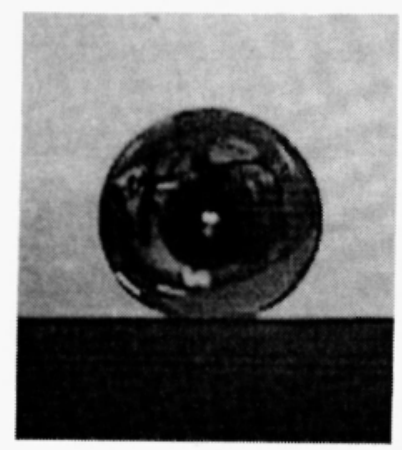

Figure 1. Shape of water droplet on the rough STA monolayer surface (volume of the droplet is $3 \mu \mathrm{L}$ ).

The static water contact angles are below $5^{\circ}$ for the surfaces of bare $\mathrm{Al}$ and $\mathrm{PEI}$ coated-Al (the boiling times of the $\mathrm{Al}$ substrate in water is 5 minutes). However, once with a STA monolayer formed, the static contact angles greatly increased to as high as about $166^{\circ}$ and the shape of the water droplet on the STA monolayer is nearly spherical (Figure 1)

\subsection{Effects of surface morphology on the wetting properties.}

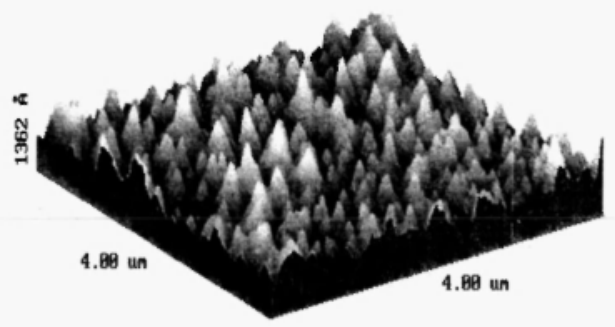

Figure 2. AFM 3D images of STA monolayers on PEI-coated polished aluminum surface with rough needle-like structure

The first step in our works is aimed at the relationship between the surface roughness and the contact angles. Surfaces of STA monolayer with different roughness are prepared by controlling the substrate boiling times in water from 0 to 5 minutes. With the surface roughness of rms increasing from $1.2 \mathrm{~nm}$ to $2.9 \mathrm{~nm}$, to 8.4 $\mathrm{nm}$, and to $19.3 \mathrm{~nm}$, the contact angles are increased from $105^{\circ}$, to $116^{\circ}$, to $148^{\circ}$, and to $166^{\circ}$, respectively. This shows that high roughness is essential to obtain the superhydrophobic surface. Figure 2 shows the superhydrophobic STA monolayer surface with contact angle of $166^{\circ}$. We can see the surface is rough (the roughness of rms about $19.3 \mathrm{~nm}$ ) and needle-like. It is well understanding, on such rough needle-like surface, that lots of crevices would be exist among the needlelike peaks. Since the STA monolayer is kind of hydrophobic material, these crevices wouldn't be wetted by water easily. Then, air would be trapped in the crevices and forming composite interface when water droplets are placed on such surface, i.e. there will be lot of air existed in the composite interface. Then, according to Cassie's equation, ${ }^{[13]}$ the fraction of air is calculated to be $96 \%$. This means that the composite interface 
is almost possessed by air and thus makes the surface extreme water-repellency.

Close relationship between the microsurface structure and the contact angle is also studied. Evaporated aluminum-coated glass is selected as the substrates and same STA monolayer surface with high roughness of rms about $20.8 \mathrm{~nm}$ is prepared. However, the contact angle for water on the surface is only about $118^{\circ}$. Figure 3(a) shows the surface AFM topography. Unlike the needle-like structure shown in figure 2 , a much different topography, which we call "mushroom-like" surface, is found. Though such mushroom-like surface possesses a high roughness, it doesn't have superhydrophobic properties. This is might attribute to that the composite interface mentioned above can't come into being in this situation due to the lack of the needle-like peaks. As roughness increased (37.6 $\mathrm{nm}$ of rms) and peak geometric structure became needle-like (Figure $3 \mathrm{~b}$ ), the surface then becomes water-repellency and give a contact angle about $162^{\circ}$. These results show that the geometric structure of surface asperities is significant for the generation of the superhydrophobic surface.
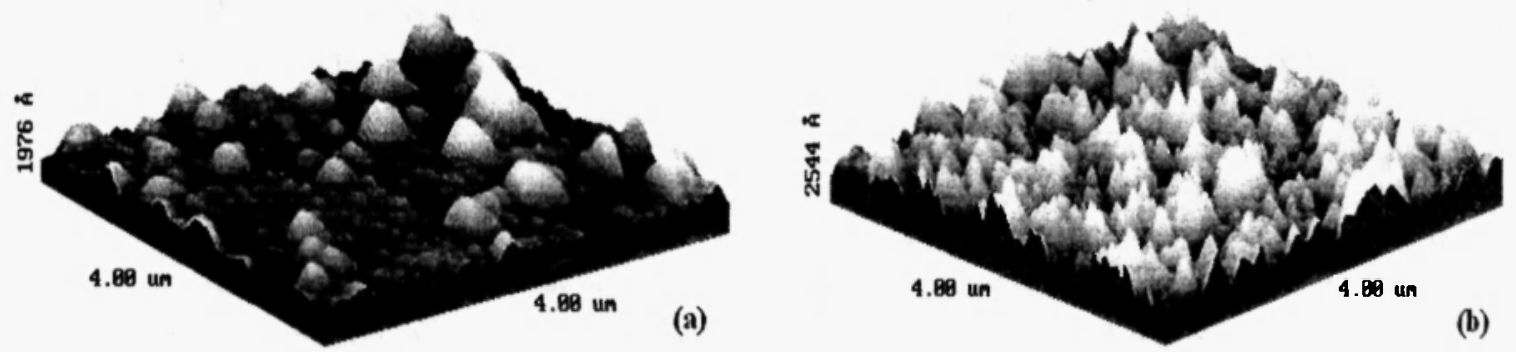

Figure 3. AFM 3D images of the ultra-thin film on evaporated aluminum film coated glass wafer with different surface geometric structure and roughness.

\subsection{The contact angle hysteresis.}

To well describe the surface wettability, the contact angle hysteresis, i.e. the difference of the advancing and receding contact angles should be considered as well. By gradually adding or removing little liquid on or from the droplet with microsyringe, we get the maximum advancing angle about $168^{\circ}$ and minimum receding angle about $156^{\circ}$. This result shows that the STA monolayer obtained in this work has a relative lower angle hysteresis of $12^{\circ}$. It has been pointed out that the contact angle hysteresis would be very small when composite interface was formed, i.e. the values of advancing and receding contact angles are tend to approach. ${ }^{[14]}$

For a further insight on the contact angle hysteresis of the STA monolayer, variation of the contact angles with times is studied also. The results are shown in figure 4 . We can see that the contact angle and the shape of the water droplet change very little at the initial evaporating long times. But it is obvious that the contact area between the water and solid surface becomes small gradually, which means that the contact line is receded with the decrease of the drop volume. Such phenomenon also reflects that the angle hysteresis is lower. However, both the contact angle and the shape of the droplet change greatly when the water becomes very little, and finally, the droplet becomes nearly film-like and the contact angle is only about $18^{\circ}$. Since water evaporating is expected to be equal to reduce the water by using microsyringe, the value $18^{\circ}$ should be the minimum receding contact angle of the STA monolayer film. This result is seemly contradictive to the minimum receding angle about $156^{\circ}$ measured by using microsyringe to reduce the water volume. We suppose that the water might penetrate into the crevices in the contact area, especially near the contact line, when the droplet is placed onto the surface keeping static for a long times (such as 1 hour). Then, composite interface changes into noncomposite interface, thus result in large angle hysteresis. 


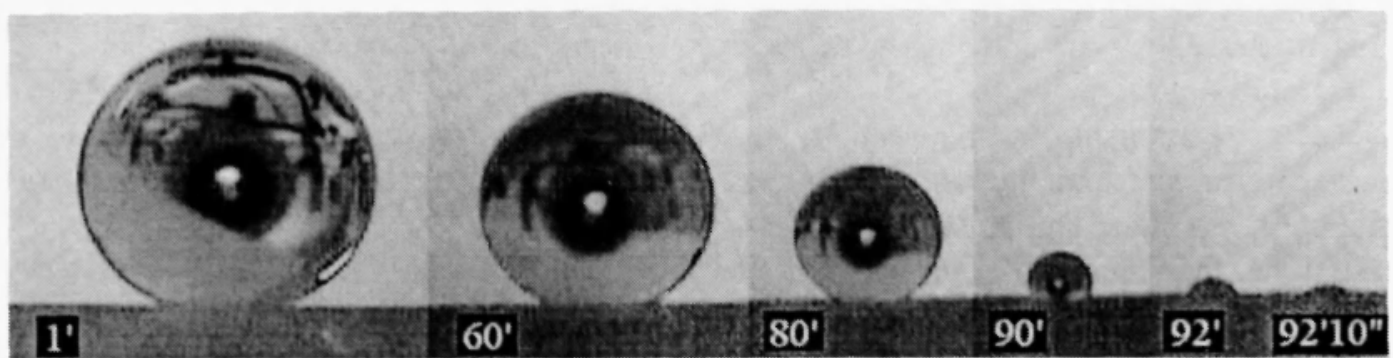

Figure 4. Variation of the water droplet shape on the ultra-thin film with times

(The initial volume of the droplet is about $4 \mu \mathrm{L}$ ).

\section{Conclusion}

A novel super water-repellent organic ultra-thin film $\left(\theta=166^{\circ}\right)$ was prepared by stearic acid chemically adsorbing onto the polyethyleneimine coated aluminum wafer. Both rough needle-like geometric structure and hydrophobic materials were essential to the formation of the super water-repellent surface. On such surface, composite interface would be formed in the contact area and which was attributed to responsible for the super water-repellent properties. The ultra-thin film has weak contact angle hysteresis with the advancing and receding contact angle about $168^{\circ}$ and $156^{\circ}$, respectively. However, if the water droplet was placed onto the surface keeping static for a long times, the contact mode might be changed (especially near the contact line area) from composite interface into noncomposite interface, and then generate large angle hysteresis.

\section{Acknowledgement}

The authors wish to acknowledge the finacial support from the Hong Kong Research Grant Council through project HKUST6155/00P and from NSFC (Grant No. 50023001) and from CAS (Grant No. KJCX-SW-L2).

The research project is also supported by the Joint Lab on Microsystems between the Hong Kong University of Science and Technology (HKUST) and the Institute of Mechanics, CAS, China, under a grant from HKUST (Project No.CMI00/01.EG02).

\section{References:}

(1) Chen, W.; Fadeev A. Y.; Hsieh , M. C.; Öner, D. Langmuir 1999, 15, 3395.

(2) Nakajima, A.; Hashimoto, K.; Watanabe, T.; Takai, K.; Yamauchi, G.; Fujishima, A. Langmuir, 2000, 16, 7044.

(3) Yang, S. Y.; Hirasaki, G. J.; Basu, S.; Vaidya, R. Journal of Petroleum Science and Engineering $1999,24,63$

(4) Bliznyuk, V. N.; Everson, M. P.; Tsukruk, V. V. Journal of Tribology 1998, 120, 489.

(5) Srinivasan, U.; Houstonm M. R.; Howe, R. T.; Maboudian, R. J. MEMS 1998, 7, 252.

(6) Rymuza, Z. Microsystem Technologies 1999, 5 , 173.

(7) Nakajima, A.; Fujishima, A. Advanced Materials $1999,11,1365$.

(8) Onda, T.; Shibuichi, S.; Satoh, N.; Tsujii, K Langmuir 1996, 12, 2125.

(9) Li, H. J.; Wang, X. B.; Song, Y. L.; Liu, Y. Q.; Li, Q. S.; Jiang, L.; Zhu, D. B. Chemical Journal of Chinese Universities 2001, 22, 759. (in Chinese)

(10) Tadanga, K.; Katata, N.; Minami, T. J. Am Ceram. Soc. 1997, 80, 3213

(11) Bahulekar, R.; Ayyangar, N. R.; Ponrathnam, S Enzyme Microb. Technol. 1991, 13, 858.

(12)Ren, S.-L.; Yang, S.-R.; Xue, Q.-J. Acta Physico-Chimica Sinica 2001, 17, 97 (in Chinese).

(13) Cassie, A. B. D. Discuss. Faraday Soc. 1948, 3, 11

(14) Wu, S. H. Polymer Interface and Adhesion; New York, 1982; Chapter 1. 